\title{
Proceedings of the 150th Semon Club, 7 December 2015, ENT Department, Guy's and St Thomas' NHS Foundation Trust, London, UK
}

\author{
Chairperson: Miss Elfy B Chevretton, Guy's and St Thomas' NHS Foundation Trust \\ Secretary: Mr Sherif Haikel, Royal National Throat, Nose and Ear Hospital, University College London Hospitals NHS Trust \\ Invited panel for pathology: Dr Ann Sandison, Charing Cross Hospital, Imperial College Healthcare NHS Trust \\ Invited panel for radiology: Dr Steve Connor, and Dr Ata Siddiqui, Guy's and St Thomas' NHS Foundation Trust \\ Professor Leslie Michaels prize for the best presentation of the meeting was awarded jointly to Lulu Ritchie for 'Diplopia and anosmia in a \\ patient presenting with sinogenic symptoms' and Ahmad Hariri for 'Multiple tumours with one unifying diagnosis'.
}

The chairperson and secretary have edited the proceedings of the meeting to reflect the discussion of each case by the expert panel and audience during the Semon Club meeting.

\section{Otology section}

Chairperson: Mr Shahzada Ahmed

Multiple tumours with one unifying diagnosis

A A Hariri, H North, S K W Lloyd

From the Manchester Royal Infirmary and Salford Royal Hospital

\section{Introduction}

Endolymphatic sac tumours are rare, malignant, locally invasive neoplasms associated with von Hippel-Lindau syndrome. These characteristics and sensitive location make them challenging to manage. We present the case of a large recurrent endolymphatic sac tumour, and discuss the diagnostic course, multi-modal management and association with von Hippel-Lindau syndrome.

Case report

A 24-year old Polish gentleman was referred with longstanding left-sided bloody otorrhoea. He had underwent excision of a left temporal bone tumour 10 years prior, with resulting VIIth cranial nerve weakness and deafness on the same side. Examination showed a large tumour filling the left ear canal, with House-Brackmann grade III facial weakness and reduced sensation on the same side. An audiogram confirmed a dead ear on the left side.

\section{Radiological findings}

Imaging revealed an extensive multi-cystic mass replacing the entire left temporal bone, in keeping with an endolymphatic sac tumour, with extension along the internal carotid artery involving the jugular bulb and cerebellopontine angle, with enhancement of the middle and posterior cranial fossa dura. An incidental finding of two cerebellar haemangioblastomas was also noted.

\section{Histological findings}

Small deposits of low-grade papillary epithelial tumour were observed on the resection specimen, within a wider area of scar and cystic degeneration, confirming the diagnosis of endolymphatic sac tumour.

\section{Management}

The patient underwent left total petrosectomy with nearcomplete removal of the endolymphatic sac tumour, neck dissection, facial-hypoglossal nerve anastomosis, and an abdominal fat graft. The patient had a good recovery and underwent post-operative radiotherapy. Genetic testing confirmed a diagnosis of von Hippel-Lindau syndrome.

\section{Discussion}

Comments were made that endolymphatic sac tumours are often hypervascular and it was asked how this was managed intra-operatively. In response, the patient underwent pre-operative balloon test occlusion and tumour embolisation to reduce vascularity. Dr Connor suggested that the patients should have imaging of the remainder of the neuroaxis to exclude a haemangioma and an ultrasound scan of the abdomen to exclude renal cell carcinoma.

Temporoparietal temporalis myofascial flap: a vascularised regional flap to reconstruct complex skull base defects following expanded endonasal approaches

A H D Silva, T H Land, S K Ahmed

From the University Hospitals Birmingham NHS Foundation Trust

\section{Introduction}

Advantages of expanded endonasal approaches to the ventral skull base (minimal manipulation of neural and vascular tissue, improved cosmesis) come at the cost of large skull base and dural defects. Reconstruction to re-establish barriers between the nasal mucosa and the subarachnoid space is critical in preventing cerebrospinal fluid (CSF) leak and ascending infection. In cases complicated by multiple surgical procedures, adjuvant radiotherapy, or tumour or iatrogenic disruption of local vasculature, standard repair with a vascularised local mucoperichondrial nasoseptal flap may not be successful. We suggest a novel modification of the vascularised regional temporoparietal fascial flap by using temporalis muscle at the tip and deeper vessels in the pedicle transposed via the transpterygoid soft tissue tunnel for a more robust repair. 
Case report

Our first patient had a recurrent pituitary macroadenoma (previously treated with an expanded endonasal approach and external beam radiotherapy), causing severe adrenocorticotropic hormone dependent Cushing's syndrome. This approach was complicated by repair-resistant (two surgical procedures) CSF leak. Our second patient underwent an expanded endonasal approach followed by proton beam radiotherapy for a clival chordoma. This approach, with debulking to prevent recurrence, was again complicated by repair-resistant (two surgical procedures) CSF leak. Successful repairs were achieved using a temporoparietal temporalis myofascial flap in both cases.

\section{Radiological findings}

Post-operative imaging at one-year follow up confirmed patency of the vascularised regional flap repair.

\section{Management}

A temporoparietal temporalis myofascial flap provided a successful skull base reconstruction in the presence of a failed local flap.

Discussion and lessons learned specifically from the meeting $\mathrm{Mr}$ Ahmed pointed out that when imaging post-operatively, the vascular flap takes up contrast, so it is important to alert the radiologist to the presence of the flap to avoid confusion with recurrent disease.

\section{Conclusion}

The vascularised regional temporoparietal temporalis myofascial flap is a robust alternative for complex skull base reconstruction for repair-resistant CSF leak when local flaps fail or are not available.

A case of multiple cranial nerve palsies, with some red herrings

D Yap, J Addams-Williams

From the Royal Gwent Hospital, Newport, Wales

\section{Introduction}

Hypoglossal schwannomas are extremely rare cranial base neoplasms, with approximately 160 cases being reported to date. These are benign, slow-growing tumours.

\section{Case report}

A 74-year-old lady presented with a 9-month history of hoarse voice, with no evidence of any ongoing infection. She had no significant past medical history and was a nonsmoker. She was found to have right-sided recurrent laryngeal nerve and hypoglossal nerve palsies.

\section{Radiological findings}

Computed tomography (CT) revealed hypertrophy of the tongue base, with some right cervical lymphadenopathy. Magnetic resonance imaging (MRI) revealed an enhancing mass within the right skull base surrounding the jugular foramen, separating the jugular vein and internal carotid arteries.

\section{Histological findings}

Biopsy of the tongue base revealed large lymphoid follicles with no germinal centres, paracortical expansion and disturbed architecture, which raised the possibility of a lymphoma.

\section{Management}

The patient's case was discussed by the lymphoma panel members, who excluded lymphoma and diagnosed a right hypoglossal schwannoma. Repeated biopsies of the right cervical lymph nodes revealed that these were benign. We discussed the option of right vocal fold medialisation. However, the patient was not keen for any surgical intervention as speech therapy had improved her symptoms. Currently, she has a yearly MRI to monitor the growth of the schwannoma.

\section{Discussion}

Dr Connor commented that the CT scan shows some bony destruction that may indicate other differential diagnoses. He suggested that an early-phase contrast CT scan could be performed to exclude a haemangioblastoma.

\section{Conclusion}

Hypoglossal schwannoma is a rare diagnosis and its management remains clinically challenging. Multidisciplinary management is highly recommended in such circumstances.

Unilateral otalgia and otorrhoea with facial nerve palsy in a patient with chronic lymphocytic leukaemia, multiple skin cancers and diabetes mellitus

\section{J R Abbas, C Williams, V Malik}

From the Blackpool Teaching Hospitals NHS Foundation Trust

\section{Introduction}

Chronic lymphocytic leukaemia causes immunosuppression. These patients are also at a higher risk of developing cutaneous malignancies, which, in chronic lymphocytic leukaemia, tend to be multiple and aggressive, with a higher incidence of post-treatment recurrence and spread. Malignant otitis externa is more common in elderly, immunosuppressed patients. We present an unusual case of an elderly patient with chronic lymphocytic leukaemia, multiple head and neck cutaneous squamous cell carcinomas (SCCs), and malignant otitis externa, with facial nerve paralysis, and discuss the dilemmas in managing this complex case.

\section{Case report}

A 78-year-old male with chronic lymphocytic leukaemia, multiple cutaneous SCCs and diabetes was admitted with worsening left-sided otalgia, otorrhoea and recent-onset, left-sided. lower motor neurone facial paralysis. He had been treated with antibiotics for recurrent ear infections by the general practitioner.

\section{Radiological findings}

A temporal bone computed tomography (CT) scan showed extensive skull base osteomyelitis. A single-photon emission CT scan showed increased uptake in corresponding areas.

\section{Histology}

Histology confirmed chronic infection with no evidence of malignancy.

\section{Management}

Because of the complexity of the case, it was managed by a team of multi-professionals, with discussions at the regional skull base multidisciplinary team meeting (MDT), skin MDT and haemato-oncology MDT. Malignant otitis externa was managed with long-term intravenous and oral broad-spectrum antibiotics and temporal bone debridement. Once malignant otitis externa was controlled, the patient received 
surface radiotherapy for the skin SCCs. Unfortunately, there was relapse of chronic lymphocytic leukaemia and the patient is currently undergoing treatment for this.

Discussion and lessons learned specifically from the meeting Some members of the faculty had come across similarly complex cases of bilateral malignant otitis externa. Dr Sandison recalled diagnosing a case of actinomycosis causing malignant otitis externa and hyperplasia of the mucosa in the paranasal sinuses.

Six years of otalgia and still itching for a diagnosis

A A Hariri, S Jayaram, S Agrawal

From the Pennine Acute Hospitals NHS Trust, Manchester

\section{Introduction}

Primary malignancies of the external auditory canal are extremely rare, with the majority being squamous cell carcinomas. Adenoid cystic carcinomas account for fewer than 5 per cent of these. These highly invasive, slow-growing tumours of disputed histogenesis often present with benign symptoms of otalgia and aural fullness, and remain undiagnosed for years. We describe the clinical history and management of a patient with adenoid cystic carcinoma of the external auditory canal.

\section{Case report}

A 47-year-old man was referred to our clinic with a 6-year history of intermittent exquisite left-sided otalgia and itchiness of the canal. Examination findings were grossly normal, other than a minimally narrowed ear canal and a $3 \mathrm{~mm}$ posterior tragal thickening with associated point tenderness. Hearing and cranial nerve examination findings were otherwise normal.

\section{Radiological findings}

Magnetic resonance imaging and computed tomography of the neck revealed thickening of the left external auditory canal extending to the bony margin, with involvement of the tragus and border of the left parotid gland.

\section{Histological findings}

Biopsy and resection specimens confirmed a diagnosis of cutaneous adenoid cystic carcinoma. This more frequently arises from the salivary glands.

\section{Management}

The patient underwent left superficial parotidectomy, lateral temporal bone resection, selective neck dissection, excision of the left pinna and reconstruction using an anterolateral thigh free flap.

\section{Discussion}

Dr Sandison agreed that cutaneous adenoid cystic carcinoma of the external auditory canal likely arises from ceruminous glands, and proposed the development of a staging system specific for primary ear malignancies. She also stated that these tumours spread rapidly to adjacent bone and lymph nodes, and 30 per cent also involve the parotid. $\mathrm{Mr}$ O'Connor pointed out that the tumour extended to the temporal bone resection margins.

\section{Conclusion}

We highlight the need to consider neoplastic causes in patients presenting with chronic ear symptoms.
Rhinology section

Chairperson: Miss Clare Hopkins

Diplopia and anosmia in a patient presenting with sinogenic symptoms

L Ritchie, C Rennie, P Clarke

From the Charing Cross Hospital, London

\section{Introduction}

The NUT midline carcinoma is a rare, malignant tumour that classically affects midline structures, including the mouth, respiratory tract and thymus. First described in 2004, it stems from the fusion of the NUT gene to a BRD4 gene to make an oncogene.

\section{Case report}

A previously healthy 41-year-old freshwater biologist presented with a 2-year history of rhinitic symptoms and headaches, in addition to a 2-month history of intermittent diplopia and anosmia. Examination revealed a nasal mass in the area of the right middle turbinate, with a right level II neck node.

\section{Radiological findings}

There was a large soft tissue mass centred in the nose and right ethmoid sinuses, extending through the floor of the anterior cranial fossa, distorting the inferior frontal lobes. The mass further distorted the orbits bilaterally, and there was perineural enhancement around the nerves in the pterygopalatine fissure and cavernous sinus.

\section{Histological findings}

The tumour was a lobular, non-keratinising, undifferentiated, high-grade primary sinonasal carcinoma. It was arising from surface endothelium, infiltrating mucosa and bone, and was present in 9 out of 10 lymph nodes. Immunostains performed in Baltimore were 90 per cent positive for NUT 1. Baltimore, in America, is the nearest centre that is able to perform this immunostain.

\section{Management}

The multidisciplinary team meeting recommendation was a combination of tumour debulking with a right level I-IV neck dissection, followed by chemoradiotherapy. This case raises awareness of an aggressive nasal tumour associated with a NUT 1 gene mutation, and highlights the importance of recognition and diagnosis. Patients should be added to the NUT midline carcinoma registry and offered inclusion in vital chemotherapeutic trials to improve survival.

\section{Discussion}

Dr Sandison pointed out that NUT midline carcinoma can be easily misdiagnosed as a sinonasal undifferentiated carcinoma.

\author{
A diagnostic conundrum in a patient with recurrent \\ epistaxis
}

F McClenaghan, C Rennie, W Grant

From the Charing Cross Hospital, London

\section{Introduction}

A patient with a history of partially resected pituitary adenoma presented with recurrent epistaxis originating from a mass on the high nasal septum. 
Case report

A 66-year-old female presented with a 6-month history of recurrent epistaxis, 9 years after an incomplete transsphenoidal resection of a pituitary adenoma without post-operative radiotherapy. Resection of the nasal mass revealed a neuroendocrine neoplasm suggestive of an olfactory neuroblastoma.

\section{Radiological findings}

Computed tomography scanning showed a definitive air gap between the mass and the cribriform plate. Positive emission tomography/computed tomography confirmed avidity in the left nasal cavity, in keeping with a neuroendocrine neoplasm.

\section{Histological findings}

The histology and immunohistochemistry findings were suggestive of an olfactory neuroblastoma; however, it was not possible to definitively exclude recurrence or extension of the pituitary adenoma. Dr Sandison mentioned that the tumour may have seeded into the nasal cavity during the first operation performed to resect the pituitary adenoma.

\section{Management}

At the multidisciplinary team (MDT) discussion, complete resection of a presumed ectopic olfactory neuroblastoma was advised. Transnasal resection with clear margins was achieved. Post-operative radiotherapy was debated and felt to offer minimal benefit for a completely resected tumour.

Discussion and lessons learned specifically from the meeting Discussion in the meeting centred on the need for post-operative radiotherapy in olfactory neuroblastoma. It was agreed that, in view of the fact that complete resection had been achieved, cranial radiotherapy would offer minimal benefit in terms of decreased recurrence when compared with its potential adverse effects.

\section{Conclusion}

An ectopic olfactory neuroblastoma in a patient with a history of pituitary adenoma but no adjuvant radiotherapy has not been previously reported. This case highlights the importance of the MDT in determining the diagnosis and best treatment in the case of rare diagnoses.

Two eyes, two masses, one diagnosis

B Silver, C Rennie, W Grant

From the Charing Cross Hospital, London

\section{Introduction}

Rosai-Dorfman disease is a rare, benign, histolytic proliferative disorder, also known as sinus histiocytosis with massive lymphadenopathy. Ninety per cent of patients are clinically well, but present with massive, bilateral, painless cervical lymphadenopathy, elevated erythrocyte sedimentation rate and hypergammaglobulinemia, with reversal of the albumin-to-globulin ratio. Extranodal manifestations are common; 20 per cent of orbital presentations are without lymphadenopathy. Aetiology is unknown, but there is speculation of a viral origin and immunological dysfunction. Treatment is controversial, as 20 per cent of patients show spontaneous recovery without intervention.

\section{Case report}

A 59-year-old male with poorly controlled insulin-dependent diabetes developed sudden onset diplopia and pain in his left orbit on waking. Clinically, there was ipsilateral proptosis, ptosis, and abducens nerve and partial ophthalmic nerve palsies, with pupillary sparing.

\section{Radiological findings}

Urgent magnetic resonance imaging of the head showed abnormal thickening and enhancement of the cavernous sinus, with anterior extension. A staging computed tomography scan and cerebrospinal fluid sampling findings were unremarkable.

\section{Histological findings}

A biopsy confirmed the presence of emperipolesis, which is a classic histopathological finding wherein red and white blood cells are phagocytosed by lymphocytes. Immunohistochemistry staining was strongly positive for S-100 and cluster of differentiation 68, but negative for cluster of differentiation 1a. Dr Sandison commented that it remains unknown if this condition is neoplastic or inflammatory.

\section{Management}

Because of a sudden decline in visual acuity, methylprednisolone and AmBisome ${ }^{\circledR}$ were started empirically. An endoscopic transnasal cavernous sinus and orbital apex biopsy changed the management. The patient was stepped down onto a reducing dose of oral prednisolone. Given the proximity of the orbital nerve, chemotherapy using methotrexate and rituximab was preferable to radiotherapy. The decision for early treatment was based on neurological involvement.

\section{Conclusion}

This rare disease is an important differential diagnosis to any malignant, infective or inflammatory lesion of the head and neck, and is made based on histological and immunohistochemistry findings.

Temporary VIth cranial nerve palsy following use of haemostatic agent in the cavernous sinus

K Senthil, A Nassimizadeh, S Ahmed

From the Queen Elizabeth Hospital, Birmingham

\section{Introduction}

FloSeal ${ }^{\circledR}$ is a flowable haemostatic matrix. It is made from bovine-derived gelatine granules mixed with freeze-dried, powdered human thrombin and sodium chloride solution. This case involved a temporary VIth cranial nerve palsy associated with the injection of FloSeal into the cavernous sinus during pituitary surgery.

\section{Case report}

A 54-year-old Caucasian male had a medically resistant macroprolactinoma with atypical radiological features. He underwent endoscopic transsphenoidal biopsy to confirm the diagnosis. Profuse intra-operative bleeding from the left cavernous sinus was controlled successfully with FloSeal. Post-operatively, the patient developed diplopia from a left abducens nerve palsy, which later resolved.

\section{Radiological findings}

Pre-operative magnetic resonance imaging demonstrated an expansile locally invasive heterogeneous lesion centred at the left petroclival space. This extended anterosuperiorly into the cavernous sinus and sella turcica, displacing the normal pituitary to the right.

\section{Histological findings}

Tissue biopsy confirmed prolactinoma. 


\section{Management}

Post-operatively, the palsy was managed conservatively, using prism glasses, with complete recovery in six weeks. This coincides with the manufacturer's timeline of FloSeal resorption.

\section{Discussion}

During discussion of the case in the meeting, Miss Hopkins asked why haematoma was excluded as a cause. Although remotely possible, a significant haematoma is considered unlikely to form in this low-flow region from minor iatrogenic trauma.

\section{Conclusion}

FloSeal, in this confined space, was considered the likely cause of the post-operative diplopia. Furthermore, corrective ophthalmic surgery was not required for complete recovery.

\section{An unusual cause of unilateral nasal obstruction}

A Goomany, S Prowse, I Smith

From the Bradford Teaching Hospitals NHS Foundation Trust

\section{Introduction}

Intranasal lesions have a wide differential diagnosis and may present a diagnostic challenge. We present the fourth known case of a rare pathology in an unusual anatomical location.

\section{Case report}

A 47-year-old lady presented following resection of a frontal lobe meningioma. Pre-neurosurgical computed tomography (CT) identified a left nasal cavity mass. She had a twoyear history of left-sided nasal obstruction, in the absence of any other symptoms or history of trauma. Nasendoscopy revealed a firm, smooth, obstructing mass arising from the left inferior turbinate, with normal mucosal covering.

\section{Radiological findings}

The CT scans demonstrated left inferior turbinate bony hypertrophy, measuring $15 \mathrm{~mm}$ in diameter, with a ground glass appearance. Magnetic resonance imaging with gadolinium revealed the lesion to be diffusely avidly enhancing. It was thought the lesion represented fibrous dysplasia; hence, a whole-body single-photon emission CT scan was performed. However, this was inconclusive as the lesion demonstrated only faint uptake.

\section{Histological findings}

Dr Sandison demonstrated dilated vascular spaces interwoven between the bony architecture of the turbinate. The covering epithelium was respiratory in nature, without dysplasia.

\section{Management}

The mass contiguous with the left inferior turbinate was resected endoscopically. Histological examination confirmed an intraosseous cavernous haemangioma.

\section{Discussion}

Dr Connor noted the sunburst pattern of trabeculations on CT imaging, which is suggestive (but not pathognomonic) of the diagnosis.

\section{Conclusion}

Intraosseous cavernous haemangiomas should be considered in patients with unilateral bony nasal cavity lesions. Surgery has a key role in both the diagnosis and management of these rare lesions.
Head and neck section

Chairperson: Mr Ricard Simo

Primary mediastinal seminoma presenting as a neck lump in a young male

L Ritchie, D Al-Okati, M Dilkes

From the Barts Health NHS Trust, London

\section{Introduction}

Primary malignant germ cell tumours can arise in any extragonadal germ cell tissue, including the peritoneum and mediastinum, the metastases of which can be the first clinical presentation. To date, there has been only one reported instance of primary mediastinal seminoma presenting as a cervical mass, with no presence of gonadal disease.

\section{Case report}

A young Caucasian male presented with a four-month history of a left lateral neck mass. Following fine needle aspiration (FNA) and further excision biopsy, histology and imaging showed this to be a metastatic deposit from a primary anterior mediastinal seminoma, with no gonadal disease. The patient was treated with Carboplatin AC10 as a single agent, with serial positive emission tomography scan monitoring.

\section{Radiological findings}

Ultrasound scans of left level IV and Vb cervical lymphadenopathy suggested metastatic disease. Thyroid, salivary gland and testicular ultrasound scans were unremarkable. Positive emission tomography/computed tomography showed a fludeoxyglucose mediastinal focus, with nodal involvement in the left neck, supraclavicular and axillary regions.

\section{Histological findings}

Initial FNA showed malignant tumour cells arranged in groups and singly, with irregular nuclear margins in a background of lymphocytes. Excision biopsy of cervical lymph nodes revealed positive findings for germ cell neoplasia markers, in keeping with metastatic seminoma of mediastinal origin.

\section{Conclusion}

Mediastinal seminomas can be primary or secondary metastatic deposits from a testicular primary. Although FNA can identify a malignant process, full histological analysis with immunohistochemistry is vital to characterise the origin and nature of a tumour in order to focus treatment.

A parathyroid mass of unknown malignant potential in an 18-year-old patient

T Visholm, V Dhar

From the William Harvey Hospital, Ashford

\section{Introduction}

Parathyroid carcinoma is a rare diagnosis, accounting for only 1 per cent of hyperparathyroidism cases.

\section{Case report}

An 18-year-old female presented with a visible left-sided neck mass. Blood tests showed raised parathyroid hormone (PTH) and adjusted calcium levels. 


\section{Radiological findings}

Ultrasound demonstrated a $6.6 \times 4.5 \times 3.7 \mathrm{~cm}$ nodular mass in close approximation with the posterior-lateral and inferior border of the left lobe of the thyroid. The Sestamibi scan findings were suggestive of a large parathyroid adenoma or carcinoma.

\section{Histological findings}

Typical features of malignancy - necrosis, extracapsular invasion and high mitotic activity - were not present. However, the large size, peripheral fibrosis and prominent blood vessels would suggest malignant disease. Dr Rosai provided an expert opinion; he diagnosed an atypical parathyroid adenoma of uncertain malignant potential.

\section{Management}

Left parathyroidectomy and hemithyroidectomy were performed. Post-operative monitoring of calcium and PTH levels will be conducted every three months for a year, biannually for a further year and then annually. Careful monitoring is required, as studies have demonstrated that 87 per cent of patients may have inadequate resection, and 25-80 per cent may have local recurrence in malignant disease cases.

\section{Discussion}

The overall opinion was to treat the patient as having malignant disease, given the uncertainty of the diagnosis. Mr Simo would have additionally performed a primary neck dissection; if needed in future, there would be increased morbidity associated with dissecting out the nerves.

The importance of a thorough history in diagnosing refractory dyspnoea

B Silver, E Hodge, G Sandhu

From the Charing Cross Hospital, London

\section{Introduction}

A 52-year-old male with a 20-year history of dyspnoea and refractory airway disease of unknown aetiology had normal lung function test results, but was unable to tolerate walking long distances. He had undergone a colectomy eight years previously for ulcerative colitis. He continued to take mesalazine and had had no recent exacerbations. Flexible nasendoscopy findings were unremarkable, apart from significant mucoid deposits in the subglottis. Microlaryngoscopy revealed multiple tracheal lesions and tracheobronchomalacia. The patient was known to suffer from nocturnal desaturations.

\section{Radiological findings}

An inspiratory and expiratory phase computed tomography neck and thorax examination with contrast revealed a reduction in the calibre of the trachea on the expiratory scans, but this was not sufficient to fulfil the criteria for dynamic airway collapse. The proximal right bronchus was tortuous and narrowed. There was a 50 per cent reduction in the left main bronchus calibre on inspiration. There was no mediastinal lymphadenopathy or parenchymal lung disease.

\section{Histological findings}

There were fragments of collagenised stroma covered by normal respiratory type epithelium, with acute and chronic inflammation, and cells including eosinophils and plasma cells.

\section{Management}

After extensive investigation to rule out relapsing polychondritis, immunoglobulin G4 sclerosing disease, amyloidosis, and granulomatosis with polyangiitis, the diagnosis of idiopathic airway disease with tracheal involvement was not changed. The patient was referred to the respiratory team for optimisation and continuous positive airway pressure treatment at night to address his nocturnal desaturations.

\section{Conclusion}

This case highlights the importance of a multidisciplinary team approach to treating a patient holistically, to try and optimise their quality of life. It is important to recognise that not all tracheal diseases are amenable to surgical intervention.

Diagnostic difficulty in a case of progressive dysphagia with chronic goitre

Y Kamhieh, H Fox, C Passant

From the Royal Gwent Hospital, Newport, Wales

\section{Introduction}

Synovial sarcoma of the hypopharynx and larynx is rare, with only 32 reported cases. Such tumours can be difficult to diagnose.

\section{Case report}

A 77-year-old woman, with a chronic goitre, presented with a hoarse voice and dysphagia. Panendoscopy showed a benign-looking aryepiglottic lesion. A biopsy was taken.

\section{Radiological findings}

Initial computed tomography (CT) scans showed a cystic mass of reported thyroid origin. Multidisciplinary review led to a repeat $\mathrm{CT}$, which identified the hypopharynx as the site of causative pathology.

\section{Histological findings}

Light microscopy showed spindle cells but was inconclusive. Immunohistochemistry confirmed a synovial sarcoma. Dr Sandison explained that the cells of origin are unknown, and not necessarily of synovial origin.

\section{Management}

Our patient had profound co-morbidities. She refused therapy, and died at home with palliation.

\section{Discussion}

Clinically, the mass appeared benign, contradicted by its rapid progression. Dr Siddiqui reviewed the first CT scan, acknowledging distortion caused by the large goitre. However, he felt the coronal reconstructions did in fact show hypopharyngeal pathology not of thyroid origin. $\mathrm{He}$ affirmed the value of magnetic resonance imaging for such cases; patient factors had prevented its use. Dr Sandison reviewed the histology, confirming the poor specificity of spindle cells, and therefore the limited value of simple microscopy. She described the hallmarks of synovial sarcoma, such as certain epithelial markers (commonly cytokeratin and epithelial membrane antigen) and chromosome 18 translocation, found in over 90 per cent of cases.

\section{Conclusion}

In this case, the rare pathology led to a diagnostic difficulty. The learning points are that these tumours often appear clinically benign and that traditional microscopy is limited. A multidisciplinary input and a high index of suspicion are necessary. 
Asymmetrical tonsils in a 51-year-old ex-smoker: an unexpected diagnosis

D Hassan, A Walker, D Kim

From the St George's Hospital, London

\section{Introduction}

A 51-year-old female presented to clinic with a 3-month history of asymmetrical tonsils following a viral upper respiratory tract infection. The patient complained of mild discomfort only. There were no red flag symptoms, but she did have a 15 pack-year smoking history.

\section{Case report}

On clinical examination, the right tonsil was enlarged; otherwise, the full head and neck examination findings were unremarkable. The patient underwent bilateral tonsillectomy. The dissection of the right tonsil was difficult; the capsule was replaced by firm tissue adherent to the underlying muscle.

\section{Histological findings}

Histology revealed scattered histiocytic aggregates with pale cytoplasm. Immunohistochemistry was diagnostic of Langerhans' cell histiocytosis.

\section{Management}

The patient was referred to the haematology multidisciplinary team. Further imaging (computed tomography staging) and a bone marrow biopsy revealed no further disease. In this case, the patient had single-system Langerhans' cell histiocytosis, and therefore only required six-monthly haematology follow up to check for systemic symptoms.

\section{Discussion}

There are no guidelines for the management of asymmetrical tonsils. It was agreed that symptomatic tonsillar asymmetry is a strong risk factor for malignancy. It was suggested that pre-operative imaging is useful to guide management in equivocal cases.

\section{Conclusion}

The management of asymmetrical tonsils should be informed by patient history, examination findings and risk factors. It is important to consider imaging in difficult cases.

\section{Paediatric section}

Chairperson: Mr Ian Hore

\section{A six-week history of tonsillitis and quinsy}

M Walsh, L Harding

From the Broomfield Hospital, Chelmsford

\section{Introduction}

Quinsy is uncommon in young children, with an incidence of 1-2 per 10000 in those under 10 years of age. The literature suggests that young children present differently to adults. They most commonly present with fever and a neck mass, with trismus present in only 21 per cent of patients. Rhabdomyosarcoma and lymphoma are the commonest head and neck malignancies in children, and both can present with unilateral swelling in the oropharynx, mimicking quinsy.
Case report

A five-year-old boy was referred with a six-week history of tonsillitis and left-sided quinsy. The parents reported his increasing difficulty eating and drinking, with associated weight loss. He had also developed new-onset snoring, with apnoeic episodes. His symptoms had not improved with repeated courses of oral and intravenous antibiotics. On examination, the child was stertorous, but systemically well and afebrile, with no trismus or lymphadenopathy. Oral examination revealed a large, left-sided soft palate swelling. All inflammatory markers were within normal limits.

\section{Radiological findings}

There was a large post-nasal space mass, almost completely opacifying the nasal cavity and deviating the soft palate inferiorly, with extension into the skull base.

\section{Histological findings}

Histology revealed a rhabdomyosarcoma of embryonal subtype.

\section{Discussion}

Understanding the presenting features of quinsy in adults and children is vital to identifying imitative conditions such as malignancy. Furthermore, Miss Pai reminded us of the importance of reassessing a diagnosis in all patients who repeatedly fail to respond to treatment.

\section{Conclusion}

A range of malignancies can present with unilateral tonsillar swelling; hence, one should be wary of patients with atypical features of quinsy.

\section{Left neck swelling for one year}

A Jain, A Marnerides, R Simo

From the Guy's and St Thomas' NHS Foundation Trust, London

\section{Introduction}

Paediatric patients with cystic hygroma of the neck can present with upper aero-digestive symptoms. It can pose a significant surgical challenge in view of the close proximity to large vessels, the pharynx and cranial nerves.

Case report

An asymptomatic four-year-old boy presented with a oneyear history of a progressive left upper neck swelling.

\section{Radiological findings}

A magnetic resonance imaging scan demonstrated a lobulated septate cyst anterior to the sternocleidomastoid muscle and close to large vessels.

\section{Histological findings}

The cystic lesion showed microscopic components of thymic gland, consistent with a cervical thymic cyst.

\section{Management}

Complete surgical excision was performed.

\section{Discussion and lessons learned}

A lymphatic malformation such as a mature cystic hygroma is the main differential diagnosis on imaging, and this can be treated either by sclerotherapy (mainly for macrocystic lesions) or excision. Cervical thymic cysts can also be cured by complete surgical excision. 


\section{Conclusion}

Cervical thymic cysts are very rare lesions that usually present in the first decade of life.

Extensive paediatric cervical lymphadenopathy - an unusual histology

F Berwick, A Jain

From the Guy's and St Thomas' NHS Foundation Trust, London

Introduction

We present a paediatric case of Rosai-Dorfman disease and reflect upon its distinctive histological findings.

\section{Case report}

A 20-month-old, otherwise asymptomatic child presented with a 2-month history of worsening cervical lymphadenopathy. Fine needle aspiration suggested reactive lymphoid hyperplasia. An infection screen was negative. Histology from an excisional biopsy revealed Rosai-Dorfman disease.

\section{Radiological findings}

Neck ultrasound showed markedly enlarged cervical lymph nodes with abnormal echotexture and increased vascularity.

\section{Histological findings}

Histology revealed sinuses distended by the accumulation of large, pale histiocytes, with some demonstrating emperipolesis. Immunostaining showed strong S100 protein expression. These features are considered diagnostic of Rosai-Dorfman disease.

\section{Management}

The majority of cases have a benign course and are monitored. However, the disease can be unpredictable, with periods of remission and exacerbation lasting for many years. Indications for treatment include vital organ involvement, systemic symptoms or life-threatening complications. Therapies include surgical debulking, radiotherapy, systemic corticosteroids and chemotherapy.

\section{Discussion}

Dr Sandison explained the distinctive morphology of histiocytes, the presence of emperipolesis and the natural history of Rosai-Dorfman disease, wherein the clinical course can be unpredictable, but the majority of cases resolve spontaneously. Dr Sandison also advised that during excision biopsy, only tissue sufficient for diagnosis should be excised.

\section{Conclusion}

Rosai-Dorfman disease is a rare and benign disorder that commonly presents as massive cervical lymphadenopathy in children and young adults. Its aetiology remains unclear, and management varies according to severity and extranodal manifestations.

\section{A four-year-old boy with recurrent discharge, otalgia and fevers}

R Menzies-Wilson, G Wong, P Das

From the Brighton and Sussex University Hospitals NHS Trust, Brighton

\section{Introduction}

We present a rare case of a four-year-old boy with a botryoid embryonal rhabdomyosarcoma of the left middle ear who presented to Brighton University Hospital.
Case report

A four-year-old boy presented with a four-month history of recurrent left ear blood and pus discharge, otalgia, and fevers. He had received antibiotics from medical professionals on 16 occasions for presumed otitis media with superimposed otitis externa. Once referred to otolaryngology, he underwent an examination under anaesthesia of the ear and excisional biopsy of a suspicious aural polyp.

\section{Radiological findings}

Magnetic resonance imaging of the head demonstrated fluid in the mastoid, opacification of the external auditory canal, extension along the Eustachian tube and intracranial extension through the tegmen tympani.

\section{Histological findings}

Histology confirmed embryonal rhabdomyosarcoma, botryoid subtype. Dr Sandison demonstrated how the tumour grows in a polypoid fashion and retains an intact epithelium; hence, it can be misdiagnosed as a benign polyp, both clinically and histologically.

\section{Management}

Subsequent to the initial excision of the polyp, the patient was started on ifosfamide, vincristine and actinomycin D chemotherapy, with concomitant radiotherapy.

\section{Discussion}

Rhabdomyosarcoma is a soft tissue malignancy, thought to originate from embryonic mesenchymal cells of striated skeletal muscle. It is a disease primarily affecting children, and is exceptionally rare in parameningeal regions. Often misdiagnosed, advanced disease is common at the time of diagnosis.

\section{Conclusion}

A high degree of suspicion is required for such cases as diagnosis is difficult. Miss Chevretton pointed out the importance of submitting aural polyps for histological examination.

\section{Progressive dysphonia in an 11-year-old female}

N Lobo, C Rennie, A Taghi

From the St Mary's Hospital, London

\section{Introduction}

Paediatric granular cell tumours of the larynx are extremely rare and, although benign, show a propensity for recurrence.

Case report

An 11-year-old female presented with a 4-month history of progressive dysphonia. Flexible nasoendoscopy revealed a polypoid lesion on the left vocal fold prolapsing into the airway. The patient underwent microlaryngoscopy and bronchoscopy, and excision of the left vocal fold lesion, with subsequent voice improvement.

\section{Histological findings}

Histopathological examination of the lesion revealed pseudoepitheliomatous hyperplasia of surface epithelium. The stroma contained an ill-defined lesion, composed of tumour cells with granular cytoplasm (enhanced on periodic acid Schiff special stain) and bland fairly central nuclei. Immunostains showed the tumour cells were diffusely positive for S100. The Ki-67 proliferation index was less than 5 per cent. 


\section{Management}

Following the recommendation of the multidisciplinary team, the patient underwent further microlaryngoscopy and bronchoscopy. This revealed recurrence of the tumour, which was excised with a carbon dioxide laser. Subsequent microlaryngoscopy and bronchoscopy, performed four months later, did not show signs of recurrence.

\section{Discussion}

Laryngeal granular cell tumours may be mistaken for vocal fold polyps or granulomas. However, they have distinct histopathological features, thus emphasising the importance of sending specimens for histopathological examination. Treatment involves complete excision with clear margins to minimise the risk of recurrence. Miss Chevretton mentioned that previously these lesions tended to be excised using laser, but the heat caused scar tissue formation. Hence, many surgeons started to use microdebriders to remove these lesions, but care must be taken to avoid excessive tissue removal. Newer lasers have less tissue penetration and heat production, and so may improve outcomes.

\section{Conclusion}

Laryngeal granular cell tumours are typically slow-growing, and often present with symptoms of dysphonia, dysphagia or odynophagia. The diagnosis is histopathological, and treatment involves complete excision with clear margins. Longterm surveillance is recommended as these tumours can recur. 